\title{
BLOCK REPRESENTATIONS FOR CLASSES OF ISOMETRIC OPERATORS BETWEEN KREǏN SPACES
}

\author{
RUDI WIETSMA
}

Abstract. The behavior of isometric and unitary operators between Kreĭn spaces is investigated by means of block decompositions. Therefore two types of isometric operators having a block representation, so-called archetypical isometric operators, are introduced. It is shown that interesting classes of isometric operators, in particular the class of unitary operators, can be expressed as a composition of archetypical isometric operators and bounded unitary operators. As a consequence of these block representations, useful information about the behavior of the isometric operators under consideration can be obtained. In particular, some results on (the Weyl families of) (quasi-) boundary triplets are presented.

Mathematics subject classification (2010): 47A06, 47B25, 47B50, 47A56. sion theory.

Keywords and phrases: Isometric and unitary relations, block representations, Weyl function, Exten-

\section{REFERENCES}

[1] D. Alpay And J. Behrnd, Generalized $Q$-functions and Dirichlet-to-Neumann maps for elliptic differential operators, J. Funct. Anal., 257 (2009), 1666-1694.

[2] R. ARens, Operational calculus of linear relations, Pacific J. Math., 11 (1961), 9-23.

[3] T. YA. AzIZOV AND I. S. IoKHVIDOv, Linear operators in spaces with an indefinite metric, John Wiley and Sons, New York, 1989.

[4] J. Behrndt, V. A. Derkach, S. Hassi, And H. S. V. DE Snoo, A realization theorem for generalized Nevanlinna functions, Oper. Matrices, 5 (2011), 679-706.

[5] J. Behrndt, S. Hassi, H. S. V. De Snoo, And H. L. Wietsma, Square-integrable solutions and Weyl functions for singular canonical systems, Math. Nachr., 284, no. 1112 (2011), 1334-1384.

[6] J. BEHRNDT AND H.-C. KREUSLER, Boundary relations and generalized resolvents of symmetric operators in Krĕ̌n space, Integral Equations Operator Theory, 59 (2007), 309-327.

[7] J. BEHRNDT AND M. LANGER, Boundary value problems for elliptic partial differential operators on bounded domains, J. Funct. Anal., 243 (2007), 536-565.

[8] J. BogNÁR, Indefinite inner product spaces, Springer-Verlag, Berlin - Heidelberg - New York, 1974.

[9] J. W. CAlkin, Abstract symmetric boundary conditions, Trans. Amer. Math. Soc., 45 (1939), 369 442.

[10] R. Cross, Multi-valued linear operators, Marcel Dekker, New York - Basel - Hong Kong, 1998.

[11] V. A. Derkach, S. Hassi, M. M. Malamud and H. S. V. De Snoo, Boundary relations and their Weyl families, Trans. Amer. Math. Soc., 358 (2006), 5351-5400.

[12] V. A. DeRKach, S. Hassi, M. M. Malamud, and H. S. V. DE Snoo, Boundary relations and generalized resolvents of symmetric operators, Russian Journal of Mathematical Physics, 16, no. 1 (2009), 17-60.

[13] V. A. Derkach And M. M. Malamud, The extension theory of Hermitian operators and the moment problem, J. Math. Sciences, 73 (1995), 141-242.

[14] P. A. Fillmore AND J. P. Williams, On operator ranges, Advances in Mathematics, 7 (1971), 254-281.

[15] M. Lesch AND M. M. Malamud, On the deficiency indices and self-adjointness of symmetric Hamiltonian systems, J. Differential Equations, 189 (2003), 556-615. 
[16] Yu. L. Shmul'Jan, Transformers of linear relations in J-spaces, Funkts. Analiz i Prilogh., 14, no. 2 (1980), 39-44 (Russian). [English translation: Functional Anal. Appl., 14 (1980), 110-113.]

[17] H. L. Wietsma, Representations of unitary relations between Krěn spaces, Integral Equations Operator Theory, 72, no. 3 (2012), 309-344. 